\title{
ADAPTIVE LOCALIZED CONTROL OF STRUCTURE-ACTUATOR COUPLED SYSTEM USING MULTI-LAYER NEURAL NETWORKS
}

\author{
Bin $\mathrm{XU}^{1}$, Zhishen $\mathrm{WU}^{2}$, Koichi YOKOYAMA ${ }^{3}$ and Takao HARADA ${ }^{4}$ \\ ${ }^{1}$ Member of JSCE, Dr. Eng., Postdoctoral Fellow of Japan Society for the Promotion of Science (JSPS), \\ Dept. of Urban and Civil Eng., Ibaraki University \\ ${ }^{2}$ Member of JSCE, Dr. Eng., Assoc. Professor, Dept. of Urban and Civil Eng., Ibaraki University \\ ${ }^{3}$ Fellow of JSCE, Dr. Eng., Professor, Dept. of Urban and Civil Eng., Ibaraki University \\ ${ }^{4}$ Member of JSCE, M. Eng., Research Assistant, Dept. of Urban and Civil Eng., Ibaraki University \\ (Nakanarusawa-cho 4-12-1, Hitachi, Ibaraki, 316-8511, Japan)
}

\begin{abstract}
The identification and control of large-scale structures are considered to be difficult due to structural complicacy and system uncertainties. In this paper, based on the concept of localized control and neural networks, a computational algorithm for earthquake response control of large-scale structures is proposed. In this algorithm, the control-structure interaction (CSI) and actuator dynamics are considered. Moreover, electro-hydraulic actuators as practical applications are designed to simulate the proposed control algorithm. Results from the numerical simulations have shown great promise for the control of large-scale civil engineering structures with localized control using neural networks.
\end{abstract}

Key Words: artificial neural networks, localized control, active control, large-scale, control-structure interaction (CSI), actuator dynamics, electro-hydraulic actuator

\section{INTRODUCTION}

With increasing research activities in the field of structural control in recent years, a number of structural control methods have been proposed. Some of the widely used structural control methods are explained by Leipholtz, et al. ${ }^{1)}$, Soong ${ }^{2)}$ and Spencer Jr. et al. ${ }^{3),}{ }^{4)}$. Most of these control algorithms require the analysis and identification of the structural system in an explicit mathematical form. These contemporary control techniques often rely on the assumption of a good dynamic mathematical model containing identified system parameters such as mass, stiffness and damping. However, there are many factors such as uncertainties, non-linearities, and measurement noises, which are so difficult to be identified and incorporated in control loop as to result in poor mathematical models and less-effective control algorithms. The attributes of control algorithms with artificial neural networks make them potentially effective in dealing with most of these problems.

The ability of artificial neural networks to approximate arbitrary continuous function provides an efficient mechanism for identification and control of civil engineering structures. The structural dynamics can be identified by neural network in an implicit form where modeling of structural parameters such as stiffness, damping and mass are not necessary. The knowledge acquired by a neural network is stored in its connection weights, which are adaptive and can change in response to outside stimuli. Neural network does not really solve control problem in a strictly mathematical sense, but it is a method of relaxation that gives an approximate solution to the problem. At present, several kinds of neural networks have been proposed. The most widely used neural network is the multi-layer neural network, which is trained with the error back-propagation algorithm.

Numerous engineering applications of neural networks have been reported in the literature of recent years. Applications of neural networks in civil engineering have been reviewed by Ghaboussi, et al. ${ }^{5)}$, Chen et al. ${ }^{6}$, Xu et al. ${ }^{7), 9), 11)}$, Sato et al. ${ }^{10), 16)}$, Bani-hani et al. ${ }^{12)}$ 13), 14), and Kumagai et al. ${ }^{15}$.

In the research of Ghaboussi, et al. ${ }^{5)}$, a threestory frame structure is modeled as a frame structure 
with three degrees of freedom per node, and an active tendon system is used as actuator. In this study, the displacements and accelerations responses of all of the three floors have been used as inputs to the neuro-controller.

However, a civil engineering structure, especially for large-scale or complex structure, usually has a great number of degrees of freedom (DOF), it is difficult to get all of the dynamic responses and to submit those to actuators. For large-scale structures, because of the high dimensionality of the full finite element model, multiple inputs and outputs, timeconsuming computation, and complex performance criteria, it is difficult to design a control strategy to achieve desired stability, robustness and so on, there seems to be a critical need for additional research in order to develop more robust and more effective control algorithm for large-scale or complex structures.

Many model reduction methods are available for the analysis and control design of large-scale or complex structures. These techniques produce a lower order model, which is beneficial to the design for controller. On the other hand, the concepts of localized and decentralized control have been proposed for dealing with large-scale problem ${ }^{8), 9), 11)}$ In the study of Hannsen et al. ${ }^{8)}$, the concept of localized vibration control was proposed to construct a control strategy for a complex structure consisting of a plate supported by two box girders. Taking advantage of model reduction method, the state space representation of the structural dynamics model was derived, and the generalized singular linear quadratic (GSLQ) control algorithm was used to decide the control forces. By using multiple actuators and single control station the transmission of vibration from the complex structure was controlled successfully without considering the hardware limitations (such as actuator dynamics and actuator output capacity). Combining the concept of localized vibration control with multi-layer neural networks, an adaptive localized vibration control strategy for a continuous concrete bridge under centralized loads was studied by Xu et al. ${ }^{9}$. And an identification method with neural networks by the using of partly observed dynamics responses has been proposed by Sato et al. ${ }^{1(1)}$.

Although the localized control show their great effectiveness to control large-scale or complex civil engineering structures, the effects of controlstructure-interaction (CSI) and the actuator dynamics in analysis and design procedures have not been considered in those studies carried out by Hannsen et al. ${ }^{8)}$ and $\mathrm{Xu}$ et al. ${ }^{9}$. Neglecting CSI effects may produce poor or perhaps catastrophic performance of the controlled system due to the unmodeled or mismodeled dynamics of the actuator-structure interaction ${ }^{12), 13)}$. Moreover, mechanical actuators, for example, electro-hydraulic actuators, employed to control structure itself are also dynamic systems. Because the useful control signal signals which are used as inputs for actuators are electrical signals rather than control forces in control problem, those control algorithms which only can be used to decide the necessary control forces can not be put into application directly. In this paper, the control-structure interaction and the dynamics of elector-hydraulic actuators are considered to study the effectiveness of multi-layer neural network in localized control for large-scale or complex structure under earthquake excitations.

\section{EQUATIONS OF MOTION FOR STRUCTUR-ACTUATOR COUPLED SYSTEM}

\section{(1) Equations of Motion of Structure}

The motion of structure under earthquake is characterized by the following equation.

$$
M \ddot{x}+D \dot{x}+K x-I_{u} f=-M I \ddot{x}_{g}
$$

where $M, D$ and $K$ are $n \times n$ mass, damping, and stiffness matrices of structure, $n$ is the number of DOF (degree of freedom) of structure; $\ddot{x}, \dot{x}$ and $x$ are $n \times 1$ acceleration, velocity, and displacement vectors; $\ddot{x}_{k}$ is the base acceleration of earthquake; $f$ is $m \times 1$ vector describing the forces produced by actuators, and $m$ is the number of actuators; $I_{*}$ is a $n \times m$ Boolean incidence matrix, the element $i_{\mathrm{mm}}$ of matrix $I_{\text {。 }}$ equals 1 when the $n$-th DOF attaches to the $m$-th actuator, and equals 0 when the degree of freedom $n$ does not attach to the $m$-th actuator; $I$ is a $n \times 1$ identity vector.

A finite element method is used to discrete the structural model in this paper.

\section{(2) Equation of Actuator ${ }^{5}$}

In this study, electro-hydraulic actuators, which have been widely used in active structural control problems, are employed as the active control devices. It is assumed that there are $m$ actuators used to control the dynamic response of structure under earthquake excitations. The dynamics of each electro-hydraulic actuator is defined by two differential equations describing the dynamics of the valve and the ram. 


\section{a) Valve Equation}

Keeping signal signal $e_{i}$ of the $i$-th actuator constant during a sampling period, the valve flow rate $q$, of $i$-th actuator during a sampling period can be determined as follows,

$$
\begin{array}{r}
q_{i}=k_{i 1} k_{i 2}\left[1-\exp \left(\frac{-t}{\tau_{i}}\right)\right] e_{i}+q_{i 0} \exp \left(\frac{-t}{\tau_{i}}\right) \\
(i=1, m)
\end{array}
$$

where $k_{i 1}, k_{i 2}$ are the constant gains and $\tau_{i}$ is the valve's time constant.

The signal signal $e$, of $i$-th actuator is issued at the beginning of each sampling period, which is considered as the origin of time $t$ in equation (2), and the valve flow rate at the beginning of the sampling period is $q_{i 0}$.

\section{b) Ram Equation}

In the case of hydraulic actuator, a feedback path exists between the velocity of each actuator and signal input to it. The relationship among the control force $f_{i}$, the ram displacement $x_{i r}$, and the valve flow rate $q_{i}$ of $i$-th actuator can be described in a differential form as follow,

$$
q_{i}=A_{i} \dot{x}_{i r}+\frac{C_{i}}{A_{i}} f_{i}+\frac{V_{i}}{\beta_{i} A_{i}} \dot{f}_{i} \quad(i=1, m)
$$

where $A_{i}$ is the area of ram, $C_{i}$ is the coefficient of leakage, $V_{i}$ is the volume of piston, $\beta_{i}$ is the compressibility of the $i$-th actuation system. It is shown that the force applied by each actuator is dependent on the actuator velocity response, that is, the feedback interaction path is intrinsic to the dynamic response of a hydraulic actuator.

\section{(3) Motion Equation of Coupled System}

The equation of actuator and the structure's equations of motion are coupled through the displacements and the actuator forces. Displacements in some of the structural degrees of freedom are tied to the ram displacements, while the generation of the actuator forces is influenced by the displacements at those structural degrees of freedom. The relation between the displacements of all of the rams and structure displacements can be represented as follows,

$$
\dot{x}_{r}=I_{u}^{T} \dot{x}
$$

where $\left\{\dot{x}_{r}\right\}=\left\{\begin{array}{llll}\dot{x}_{1 r} & \dot{x}_{2 r} & \ldots & \dot{x}_{m r}\end{array}\right\}$

The coupled equations for the structure-actuator coupled system are obtained from equations (1), (3), (4) and (5). The left-hand side of equation (3) is the valve flow rate, which is considered as a known quantity and is computed from equation (2). Finally, the coupled equation can be derived as,

$$
\begin{aligned}
& {\left[\begin{array}{cc}
M & 0 \\
0 & 0
\end{array}\right]\left\{\begin{array}{l}
\ddot{x} \\
\ddot{f}
\end{array}\right\}+\left[\begin{array}{cc}
D & 0 \\
A I_{u}^{T} & d
\end{array}\right]\left\{\begin{array}{l}
\dot{x} \\
\dot{f}
\end{array}\right\}+\left[\begin{array}{cc}
K & -I_{u} \\
0 & k
\end{array}\right]\left\{\begin{array}{l}
x \\
f
\end{array}\right\}} \\
& =\left\{\begin{array}{c}
-M I \ddot{x}_{g} \\
q
\end{array}\right\}
\end{aligned}
$$

$$
\text { where } \begin{aligned}
q & =\left\{\begin{array}{llll}
q_{1} & q_{2} & \cdots & q_{m}
\end{array}\right\}^{T} \\
A & =\left[\begin{array}{cccc}
A_{1} & 0 & \cdots & 0 \\
0 & A_{2} & \cdots & 0 \\
\vdots & \vdots & \ddots & \vdots \\
0 & 0 & \cdots & A_{m}
\end{array}\right] \\
d & =\left[\begin{array}{cccc}
d_{1} & 0 & \cdots & 0 \\
0 & d_{2} & \cdots & 0 \\
\vdots & \vdots & \ddots & \vdots \\
0 & 0 & \cdots & d_{m}
\end{array}\right] \\
k & =\left[\begin{array}{cccc}
k_{1} & 0 & \cdots & 0 \\
0 & k_{2} & \cdots & 0 \\
\vdots & \vdots & \ddots & \vdots \\
0 & 0 & \cdots & k_{m}
\end{array}\right] \\
d_{i} & =\frac{V_{i}}{\beta_{i} A_{i}} \text { and } k_{i}=\frac{C_{i}}{A_{i}} \quad(i=1, m)
\end{aligned}
$$

The equation of structure-actuator coupled system is numerically integrated by Newmark- $\beta$ method to obtain the solution of dynamic response of structure and the coupled forces between structure and actuators under earthquake excitations and control signals. The integration time step used in the numerical analysis is chosen to be a small fraction (one-tenth) of the sampling period. This will allow for a realistic representation of the generation of actuator forces during the sampling period as a result of the actuator's dynamics, and the interaction between the structure and the actuators.

\section{LOCALIZED NEURO-CONTROLLER}

\section{(1) Concept of Localized Control}

Usually, it is difficult to control the vibration of a entire large-scale or complex structure, moreover the full finite element model is not suitable for controller design because the high-order models maybe inaccurate and the computations for the controller become too time-consuming and inefficient. Comparing with general vibration control, the aim of localized vibration control is to control only localized areas rather than the entire structure. And then deciding the position of sensors 


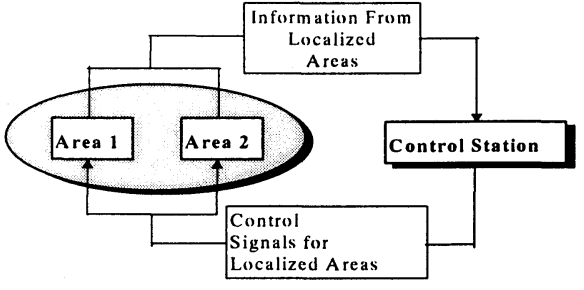

Fig. 1 Concept of localized control

and actuators is somewhat simpler in a localized vibration control problem than in a general vibration control problem. For localized vibration control, sensors and actuators are usually located within the control areas, which comprise only a small portion of the full structure. The information submitted to controller is obtained from localized areas and the control signals act in localized areas ${ }^{8), 9)}$.

The basic concept of localized control is demonstrated in Fig.1.

\section{(2) Multi-layer Neural Network}

A typical three-layer back-propagation neural network with $l$ nodes in the input layer, $m$ neurons in the hidden layer and $n$ neurons in the output layer is designed. Weights $w_{h i}(h=1, m ; i=1, l)$, $w_{o h}(o=1, n ; h=1, m)$ are used to represent the strength of connections of the neurons between the input layer and the hidden layer, the hidden layer and output layer respectively.

The first type of operation of three-layer neural network is called as "feed forward". In this operation the output of a neuron $i$ of layer $N$ can be shown as,

$$
\begin{gathered}
x_{i}=f\left(\bar{x}_{i}^{N}\right) \\
\bar{x}_{i}^{N}=\sum_{j=1}^{J} w_{i j}^{N, N-1} x_{j}^{N-1}-h_{i}^{N} \\
f(x)=\frac{1}{1+e^{(-x)}}
\end{gathered}
$$

where $f(x)$ is an activation function, which is differentiable; $x_{j}^{N-1}$ is the output of neuron $j$ of layer $N-1 ; h_{i}^{N}$ is the bias representing the threshold of the activation function of neuron $i$ of layer $N, J$ is the number of neurons in Layer $N-1$.

The second type of operation of the multi-layer neural network is called as "error back-propagation". The error function $E$ is defined as,

$$
E=\sum_{p} \sum_{i} \frac{\left(d_{i}-x_{i}^{F}\right)^{2}}{2}
$$

where $d_{i}, x_{i}{ }^{F}$ are the desired output and the output of the $i$-th neuron in output layer respectively; $i, p$ are the number of output neurons of output layer and the total number of patterns (examples) contained in the training set.

Usually, the widely used learning algorithm for training neural networks is delta rule which is based on the gradient steepest decent method. In order to increase the rate of learning and yet avoid the danger of instability, a modified algorithm called the generalized delta rule is used in this paper by including a momentum term, which describe the relationship of the correction of weight $w_{i j}^{N, N-1}$ between layer $N-1$ and layer $N$ at iteration $k+1$ and it at iteration $k$ as follows,

$$
\Delta w_{i j}^{N, N-1}(k+1)=\eta \delta_{i}^{N} x_{j}^{N-1}+\alpha \Delta w_{i j}^{N, N-1}(k)
$$

where

$$
\delta_{i}^{N}=-\frac{d E}{d \bar{x}_{i}^{N}}
$$

$\triangle w_{i j}^{N, N-1}(k+1)$ and $\triangle w_{i j}^{N, N-1}(k)$ are the correction applied to weight $w_{i j}^{N, N \cdot 1}$ at iteration $k+1$ and $k ; \eta$ is a positive constant called the learning-rate parameter, and $\alpha$ is usually a positive value called the momentum constant. In any event, care has to be exercised in the selection of the learning-rate parameter. A small learning-rate parameter lead to a slower rate of learning, on the other hand, if we make the learning-rate parameter too large, the learning procedure may become unstable. In this paper, let $\eta=0.8$. Moreover, as described by Hagiwara $^{17)}$, the momentum constant must be restricted to the range $0 \leqq|\alpha|<1$, here let $\alpha=0.6$ here.

The updated value of weight $w_{i j}^{N, N-1}$ at iteration $k+1$ is computed as follows:

$$
w_{i j}^{N . N-1}(k+1)=w_{i j}^{N . N-1}(k)+\Delta w_{i j}^{N . N-1}(k+1)
$$

The neural network learning process is to adjust the connection weights by repeatedly training thereby minimizing the error between the network output and the desired target in the training set. 


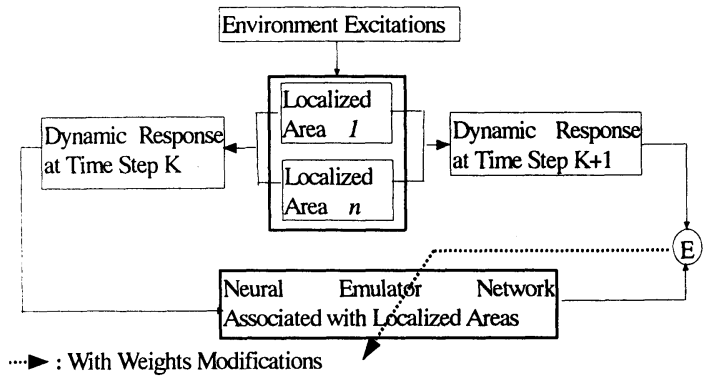

Stage 1: Localized Identification

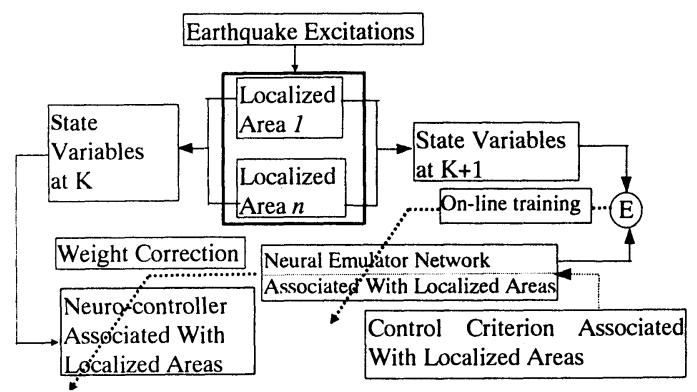

Stage 2: Localized Control

Fig. 2 Basic idea of the localized control using neural networks

\section{(3) Localized Control Using Neural Network}

a) Architecture of Localized Control with Multi-layer Neural Networks

In this paper, two typical three-layer back-propagation neural networks are adopted as neural emulator network and neuro-controller respectively. The basic idea of the localized control with multi-layer neural network is illustrated in Fig.2. It includes two stages: localized identification and controller training for the localized areas of structure and control signals calculating.

In the first stage, the localized areas of the structure-actuator coupled system are identified by the method of training the neural emulator network. In the second stage, based on the trained neural emulator network, a neuro-controller is trained to generate control signals for localized areas.

\section{b) Localized Identification for Localized Areas}

The training process of neural emulator network is to establish the appropriate connection weights between neurons of each layer by a form of supervised learning with the help of training set which is composed of a number of patterns of network inputs and desired network outputs of localized areas.

Based on the training algorithm described above, the neural emulator network is off-line trained. The weights are initialized with small random values

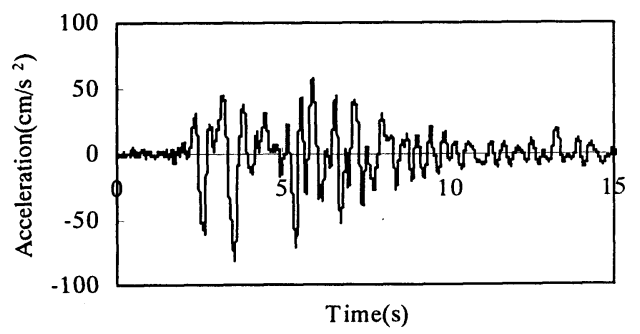

(a) Kobe earthquake record with $10 \%$ amplitude

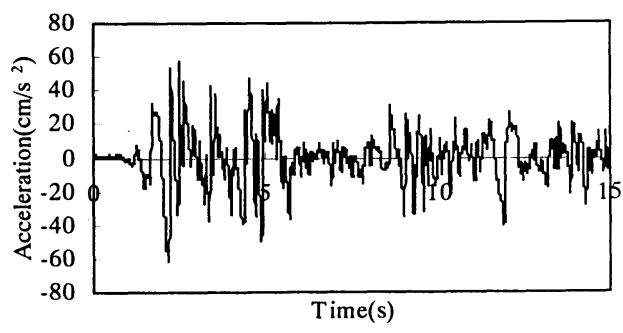

(b) El-Centro earthquake record with $20 \%$ amplitude Fig. 3 Earthquake records

first. The outputs are then computed by feeding forward the inputs through the network. The error function is calculated from the difference between the outputs of neural emulator network and the dynamic response recorded by sensors in the localized areas of the structure-actuator coupled system. By back-propagating the error function to adjust the weights, the neural emulator network can be trained to achieve a desired accuracy for modeling the dynamic behavior of localized areas of the structure-actuator coupled system.

c) Training of Neuro-controller and Calculating Control Signals for Localized Areas

The neuro-controller is trained based on the neural emulator network trained above.

At first, the error of signals can be decided by back-propagating the error function $E$ through the trained neural emulator network without changing the weighs. And then the error of signal is back-propagated to adjust the weights of the neuro-controller. This training process is repeated until the structural responses satisfy the control criterion.

In this paper, the first 15 -second record of Kobe earthquake (Hyogo-ken Nanbu Earthquake, Jan. 17, 1995) with $10 \%$ of the amplitude is used to train the neuro-controller. In order to study the adaptability and robustness of the trained neuro-controller, the effectiveness of the trained neuro-controller is shown in this paper when structure-actuator coupled system is subjected to Kobe earthquake record with $10 \%$ and $20 \%$ amplitude and the first 15 -second El-Centro earthquake record (Imperial Valley, May 


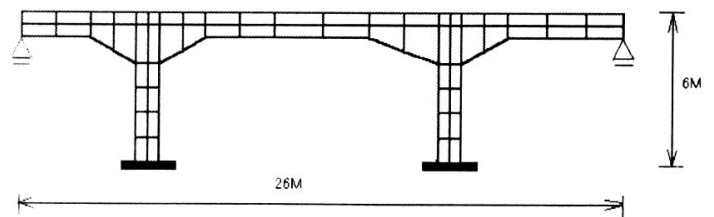

Fig. 4 FEM mesh of structure

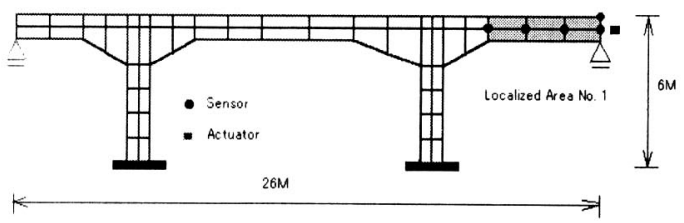

(a) Case 1

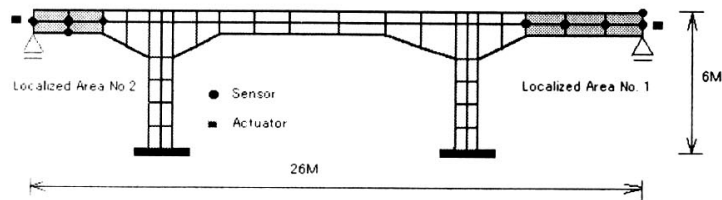

(b) Case 2

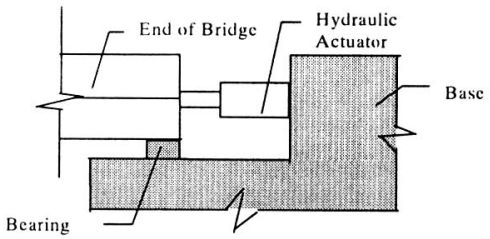

(c) Connection of Actuator and Structure

Fig.5 Coupled system of bridge structure and actuator

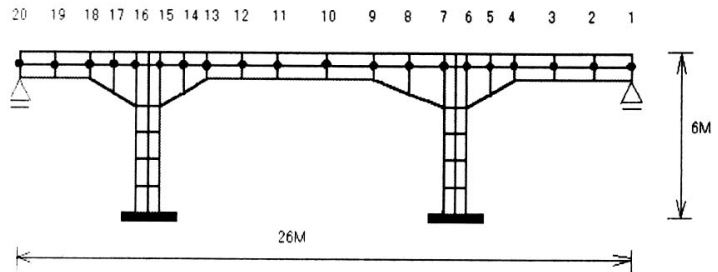

Fig. 6 Evaluation points

19, 1940) with $20 \%$ amplitude. The earthquake excitations used are shown in Fig.3. The sampling period of the earthquake excitations is $0.05 \mathrm{~s}$.

\section{NUMERICAL SIMULATIONS}

\section{(1) Design of Actuator}

The following parameters of electro-hydraulic actuators are used for composing the equations of the actuator dynamics.

$A=$ area of ram, $500 \mathrm{~cm}^{2} ; V=$ volume of chamber, $5000 \mathrm{~cm}^{3} ; C=$ coefficient of leakage, $0.1 \mathrm{~cm}^{5} /($ kgf s$)$; $\beta=$ compressibility of actuator, $2.1 \mathrm{e}^{5} \mathrm{kgf} / \mathrm{cm}^{2} ; \tau=$ time constant, $0.20 \mathrm{~s} ; u_{\max }=$ maximum absolute value of actuator force, $80 \mathrm{tf}, k_{1}=$ actuator gain, $10.0 ; k_{2}=$ actuator gain, $10.0 \mathrm{~cm}^{3} / \mathrm{s}$; and $k_{3}=$ actuator transducer gain, $100.01 / \mathrm{kgf}$. In this paper, two actuators are considered to be the same type.

The actuator signals are generated by neuro-controller at the beginning of each sampling period and are kept constant within each sampling period. This will allow for the analysis to properly account for the effects of actuator dynamics in generating actuator forces.

\section{(2) Structure-Actuator Coupled System}

In order to demonstrate the performance of the neuro-controller for the control of large-scale or complex structures under earthquake, a plane structural model of a continuous concrete bridge is adopted in the analysis. FEM mesh is shown in Fig.4. As material properties, Young modulus of concrete $E_{c}=2.1 * 10^{5} \mathrm{kgf} / \mathrm{cm}^{2}$, density of concrete $\rho=2.5 \mathrm{t} / \mathrm{m}^{3}$, poisson ratio of concrete $\gamma=0.166$, and damping coefficient is set to be 0.02 .

As illustrated in Fig.5 (a) and (b), two cases corresponding to two kinds of control strategies are investigated in this study. In case 1 , only one actuator corresponding to one localized area is employed for the control of the structure, while in case 2, there are two actuators corresponding to two localized areas adopted to control horizontal displacement response. The displacements and accelerations in horizontal direction (X direction) and vertical direction ( $\mathrm{Y}$ direction) at the position of sensors in each localized area are used as parts of inputs to neural emulator network and neuro-controller. Control forces act in horizontal direction. Connection between structure and hydraulic actuator is illustrated in Fig.5 (c). In order to manifest results of simulation, 20 points shown in Fig.6 are used as the evaluation points.

\section{(3) Localized Identification for Localized Areas}

The training of the emulator-emulator network is a deliberate and challenging process, because it is the basement of the control loop. The architecture of the three-layer neural network used as neural emulator network is presented in Fig.7.

The input layer includes the displacements and accelerates in horizontal and vertical direction from sensors in each localized area, actuator signals and earthquake record at time step $K$. The number of neurons in hidden layer is set to be two times of those in input layer. The neurons in output layer represent the forecast horizontal and vertical displacement responses at the position of sensors in localized areas at time step $K+1$. In case 1 , the input 


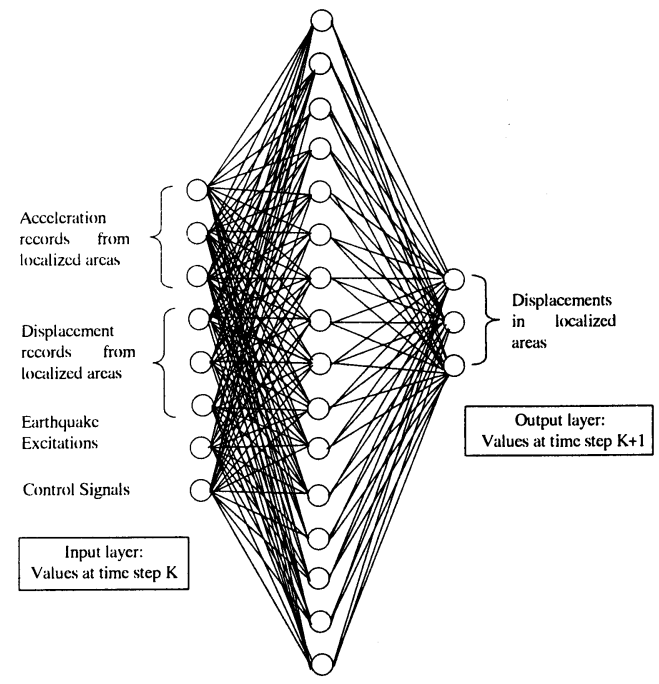

Fig.7 Neural emulator network

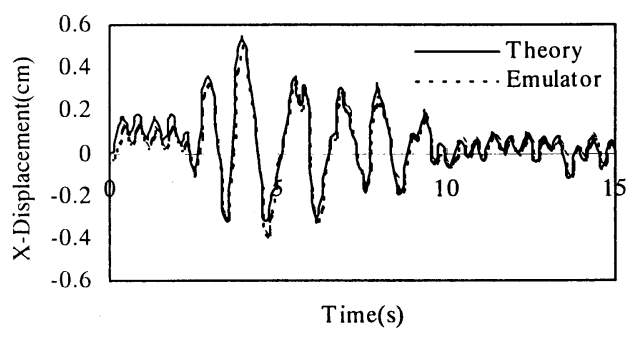

(a) Evaluation Point 1

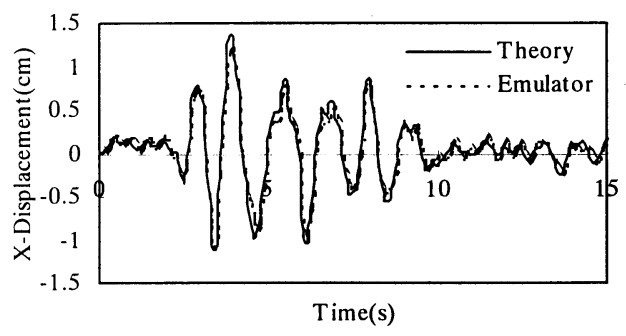

(b) Evaluation Point 4

Fig. 8 Comparison between displacement response produced by neural emulator network and calculated by FEM in case 1

layer includes 22 neurons, and in case 2, it includes 38 neurons. The number of neurons in output layer of case 1 and case 2 is, respectively, 10 and 18 .

The training data sets for the purpose of training neural emulator network are constructed from the numerical integration analysis results while the structure-actuator coupled system is subjected to random control signals and the first 15 -second Kobe earthquake record with $10 \%$ amplitude. The numerical integration analysis is carried out with an

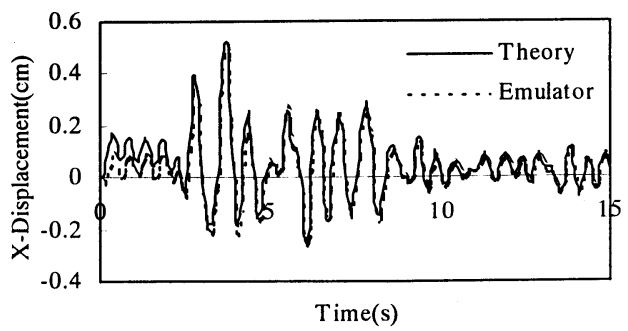

(a) Evaluation Point 1

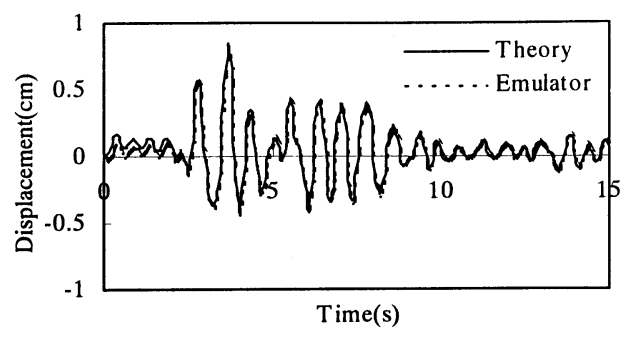

(b) Evaluation Point 2

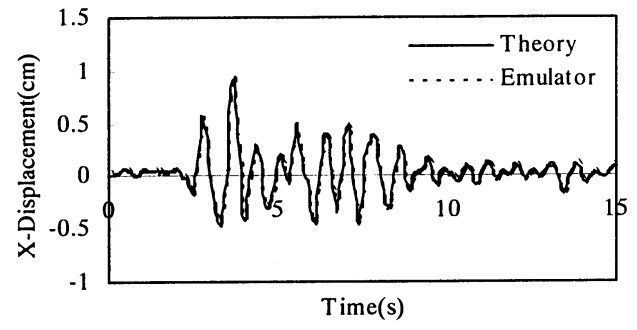

(c) Evaluation Point 18

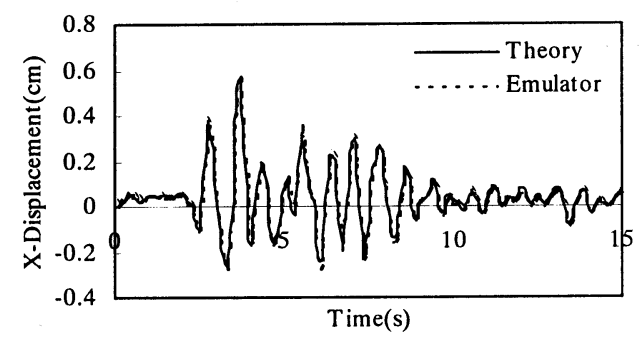

(d) Evaluation Point 20

Fig.9 Comparison between displacement response produced by neural emulator network and calculated by FEM in case 2

integration time step of $0.005 \mathrm{~s}$. The training data sets are performed with the data taken at the intervals of the sampling period of $0.05 \mathrm{~s}$.

By means of the learning rule described above, the training data sets performed above are enough to train the neural emulator network in order to model the dynamics of the coupled system and to generate the dynamic responses of structure. Fig.8 and Fig.9 give the comparison between the displacements 
response determined from the numerical integration analysis by FEM and those forecast by the trained neural emulator network when the structure-actuator coupled system subjected to Kobe earthquake record with $10 \%$ amplitude and random control signals between 0.1 and -0.1 in case 1 and in case 2 , respectively.

This indicates that the trained neural emulator network has learned about the dynamic behavior of the structure-actuator coupled system and is capable of predicting the dynamic response in localized areas. In other words, information from localized areas is enough to predict dynamic responses by multi-layer neural network.

In order to demonstrate the ability of learning of the multi-layer neural network adopted as emulator network, Fig.10 presents the relationship between the average relative error and the times of iteration of learning procedure. The average relative error is defined as follows:

$$
e=\frac{\sum_{i=1}^{I} \sum_{j=1}^{J}\left|x_{i j}-y_{i j}\right|}{\sum_{i=1}^{I} \sum_{j=1}^{J}\left|y_{i j}\right|}
$$

where $I, J$ are the total number of patterns of training set and the total number of output neurons of output layer of neural emulator network, and $x_{i j}, y_{i j}$ is , respectively, the output of neural emulator network and the theory value from integration.

The result shows that the average relative error can be guaranteed bellow $0.2 \%$ and neural emulator networks in both case 1 and case 2 can be used to predict the response of coupled system with the same precision.

Fig.11 demonstrates the consumed time for training neural emulator network in two cases, it is shown that the training procedure is more time-consuming in case 2 than it in case 1 because the scale of neural emulator network in case 2 is greater than it in case 1 . Therefore, the concept of localized control using multi-layer neural network is advantageous to the realization of on-time control because the training procedure of a small-scale neural network is less time-consuming.

\section{(4) Training of Neuro-controller}

A three-layer neural network called as neurocontroller is proposed for control. Fig.12 indicates the architecture of neuro-controller.

The inputs to neuro-controller include the displacements and accelerations in horizontal and vertical direction from sensors in localized areas, earthquake excitations and the control signals at the last time step $K$. In case 1, the input layer includes 22 neurons, while in case 2 , it includes 38 neurons.

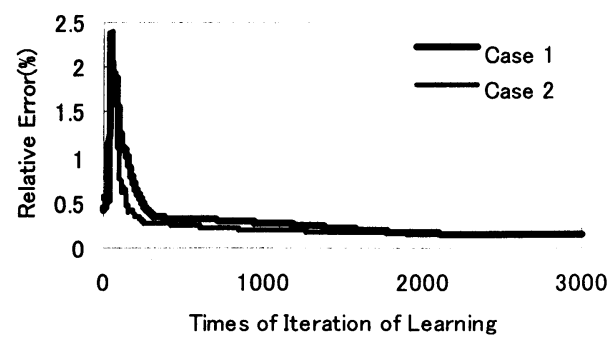

Fig.10 Relationship between average relative error and the times of iteration of learning

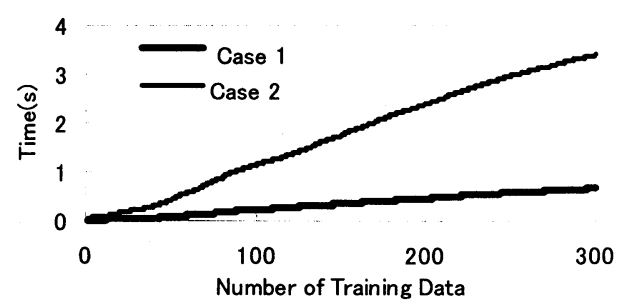

Fig. 11 Time used to train emulator-network

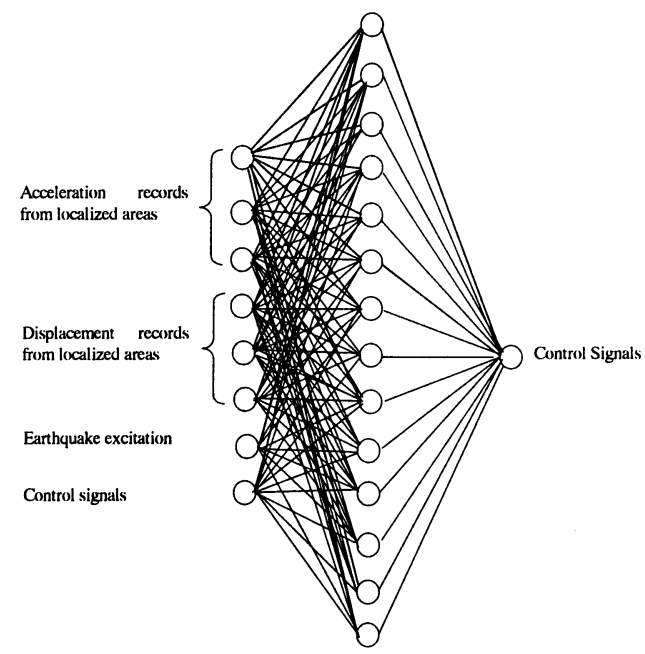

Fig.12 Architecture of Neuro-controller

The number of neurons in hidden layer is set to be two times of those in input layer. The number of neurons in output layer, which represent the necessary control signals at the current time step, is equal to the number of actuators employed. So there is one neuron, 2 neurons in output layer in case 1 , respectively, in case 2 . Taking advantage of the strategy described above, the neuro-controller can be trained and used to calculate the necessary control signals for each actuator.

The control criterion defines the objective of control. A realistic objective of structural control is to reduce, but not completely eliminate the motion of structure under external loads. It is difficult to re duce the structure response to zero, a weak control 


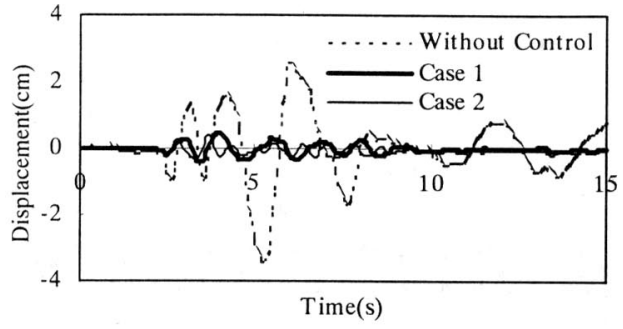

Fig. 13 Time history of displacement response in localized Area 1 subjected to Kobe earthquake with $10 \%$ amplitude with and without neuro-controller

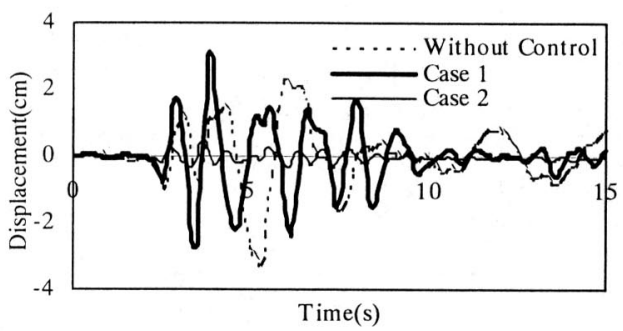

Fig.14 Time history of displacement response in localized area 2 subjected to Kobe earthquake with $10 \%$ amplitude with and without neuro-controller

criterion may be effective. In this study, the control criterion is set to eliminate the displacements in horizontal direction of the control points to a small value of $0.5 \mathrm{~cm}$. And the maximum capacity (maximum of the possible control force) of the actuators are defined as 80 tf. A neuro-controller is trained with this control criterion and is applied to the control of the structure-actuator coupled system.

\section{(5) Analysis of Simulation Results}

The performance of the trained neuro-controller is illustrated through numerical simulations. As the first example, the trained neuro-controller is exploited to control the structure under the Kobe earthquake record with $10 \%$ amplitude, which is the same earthquake record as that used in the training of the neuro-controller.

Fig.13 and Fig.14 show the comparison of time history of displacements on control point with and without neuro-controller in area 1, respectively, in area 2. From Fig.13, it can be seen that displacement response in localized control area 1 where an actuator is located can be controlled successfully basing on the concept of localized control using neural network both in case 1 and in case 2. Fig.14 shows that control effect of displacement response in localized area 2 in case 1 is not as good as it in case 2 because there is no actuator in localized area 2 in case 1 , however, the

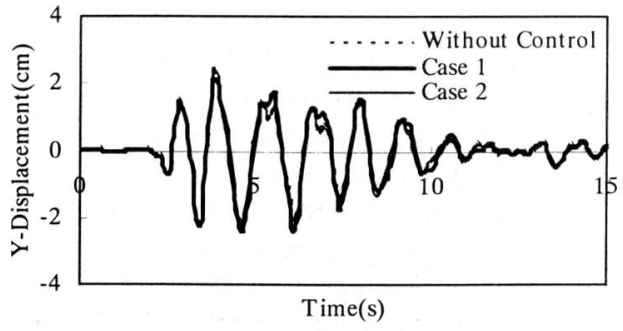

Fig. 15 Comparison of displacement in vertical direction of point 11

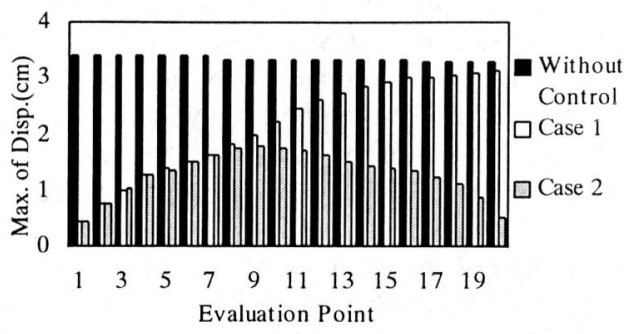

(a) Horizontal displacements

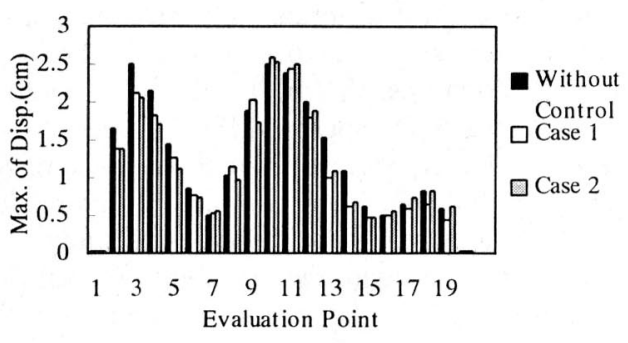

(b) Vertical displacements

Fig. 16 Comparison of maximum of displacement

displacement response in localized area 2 can be controlled successfully by the actuator located there in case 2.

This demonstrates that neuro-controller can decide the necessary control signals based on the information from localized areas. The results show that the trained neuro-controller has learned to control the displacement of control point and has been able to realize the control criteria. These results suggest that neural-network-based localized vibration control may be a practical method for large-scale structure system.

The displacement response in vertical direction of point 11 in the middle of the span is illustrated in Fig.15. It can be found that actuators in horizontal direction do not magnify the displacement response in vertical direction in the middle of the span of the structure. In order to investigate the control effectiveness of each actuator to entire structure, the comparison of maximum of the displacement responses in horizontal and vertical direction on 
evaluation points in the entire beam part of the structure with and without control are indicated in Fig.16. As shown in Fig.16 (a), it can be seen that horizontal displacements in localized area 2 is partly controlled by the actuator located in localized area 1 , but it is impossible to control the localized area 2 of the structure to a desired level with a actuator in localized area 1. Moreover, because there are no actuators used to produce control force in vertical direction, it is demonstrated in Fig.16 (b) that the displacements in vertical direction cannot be controlled. In spite of this, it is found that the vertical displacement at any node is not amplified due to the horizontal actuators. Because there is not any basement used to support a hydraulic actuator directly in the middle of the span, it is difficult to produce control force directly by hydraulic actuator. In order to control the vibration in vertical direction in the middle of the span, another kind of actuators such as active mass drivers (AMD) may be used. By virtue of the motion of AMD, control force can be produced both in horizontal and in vertical direction according to control criterion. In order to realize the full potential of neuro-control method for large-scale structures, the localized control method for Active Mass Damper (AMD) is need to be studied with the consideration of the characteristics of actuator and the control-structure interaction (CSI). This is beyond of the scope of this study.

In order to investigate the possibility of carrying out on-time control, the consumed times for the purpose of on-line training neural emulator network, neuro-controller and deciding control signals at each sampling step by neural-controller in case 1 and case 2 are shown in Fig.17. The simulations are carried out on a VT-Alpha 533 workstation with a RAM of $128 \mathrm{MB}$ and the CPU with speed of $533 \mathrm{MHz}$. It can be seen that the consumed time of each sampling step in case 2 is greater than it in case 1. Neural network with small scale is beneficial for carrying out on-time control. In spite of this, the largest consumed time in each sampling step in case 2 is far less than the sampling period of 0.05 second, therefore on-time control can be carried out by multi-layer neural network even in the case 2 .

In this investigation, signals $e_{i}(i=1, m)$, which are electrical signals, are determined directly by the trained neuro-controller and can be used as inputs to actuators to determine the flow rates $q_{i}(i=1, m)$ of the corresponding hydraulic actuators. The coupled forces between actuators and structure are implicit variables, which cannot be determined by neuro-controller directly. In the studies of Hannsen et al. ${ }^{8)}$ and $\mathrm{Xu}$ et al. ${ }^{9}$, the control forces were calculated by controller directly, however, because the CSI and dynamics was not considered, the

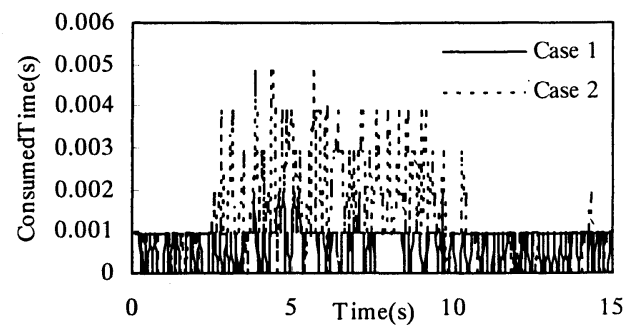

Fig. 17 Consumed times to on-line train emulator network and to decide control signal in each sampling step

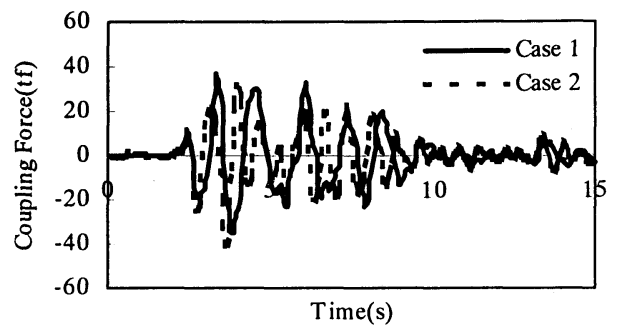

(a) Coupled force between actuator 1 and structure

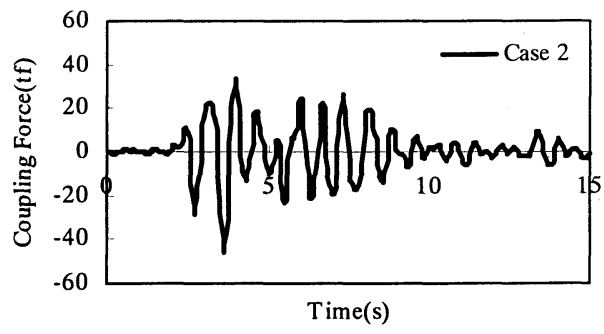

(b) Coupled force between actuator 2 and structure

Fig. 18 Coupled force between actuators and structure

useful control signals rather than control force can not be determined directly. In order to realize and implement those innovative control strategies for dynamic hazard mitigation, the CSI and dynamics should be considered.

The coupled forces between actuators and structure, when the structure was subjected to Kobe earthquake record with $10 \%$ amplitude, are shown in Fig.18. It can be found that the control force is less than the actuator capability. The supposed abilities of actuators are enough to control the vibration under Kobe earthquake record with $10 \%$ amplitude

\section{(6) Discussion on Adaptability of Localized Neuro-controller}

In the previous example the structure-actuator coupled system is subjected to the Kobe earthquake with $10 \%$ amplitude, which is the same earthquake 


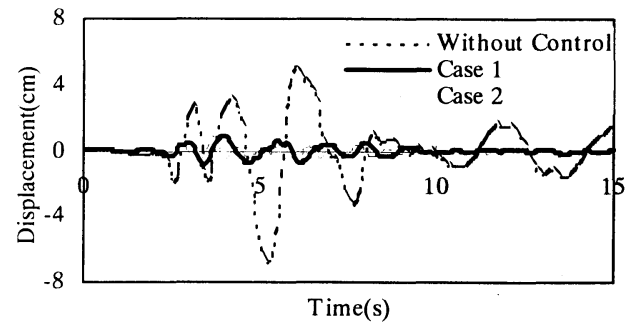

Fig. 19 Time history of displacement responses in localized area 1 subjected to Kobe earthquake with $20 \%$ amplitude and controlled with neuro-controller trained on Kobe earthquake with $10 \%$ amplitude

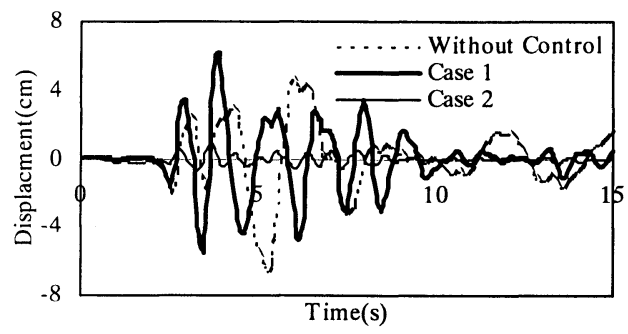

Fig. 20 Time history of displacement responses in localized area 2 subjected to Kobe earthquake with $20 \%$ amplitude and controlled with neuro-controller trained on Kobe earthquake with $10 \%$ amplitude

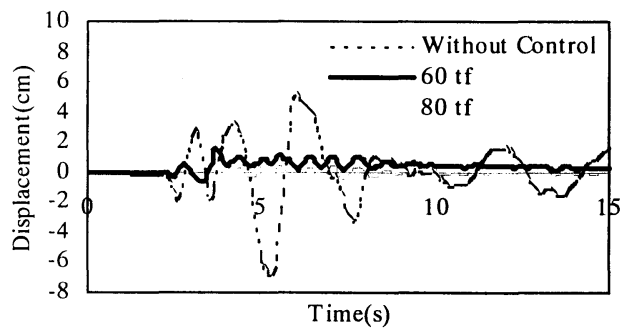

Fig.21 Influence of actuator capability

record as that used in training of the localized neuro-controller.

In the next example, we explore the performance of localized neuro-controller when the structure-actuator coupled system is subjected to ground shaking with high intensity. The localized neuro-controller, which is trained with the Kobe earthquake record with $10 \%$ amplitude, is employed in this simulation too. This localized neuro-controller is applied to control the structure-actuator coupled system that is subjected to the Kobe earthquake record with $20 \%$ amplitude. The results of the analyses are shown in Fig. 19 and Fig.20. It can be found that the trained localized

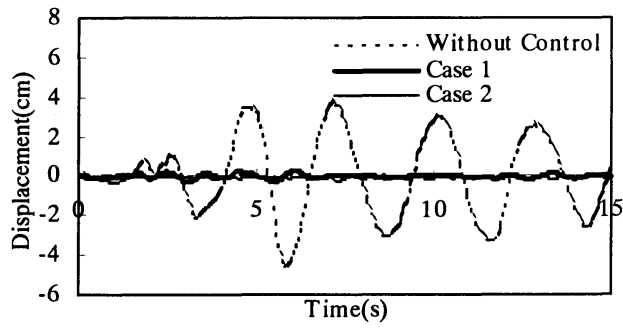

Point 1 (control point)

Fig. 22 Time history of displacement response of in localized area 1 subjected to El-Centro earthquake record with $20 \%$ amplitude and controlled with neuro-controller trained on Kobe earthquake record with $10 \%$ amplitude

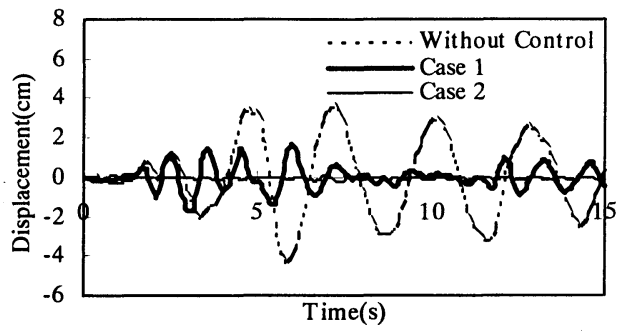

Point 20 (control point)

Fig. 23 Time history of displacement response in localized area 2 subjected to El-Centro earthquake record with $20 \%$ amplitude and controlled with neuro-controller trained on Kobe earthquake record with $10 \%$ amplitude

neuro-controller has done its best to control the displacement response too.

And the issue of the effect of the actuator saturation is considered here. Fig.21 gives the time history of displacement response on the control point in localized area 1 of the structure-actuator coupled system subjected to Kobe earthquake record with $20 \%$ amplitude and controlled by neural-controller when actuators with different saturation capabilities of 60 tf and 80 tf are used respectively. It can be found that the maximum capability of actuators influences the control efficiency, but the neural-controller does its best to control the structure-actuator coupled system.

This demonstrates that the performance of the neuro-controller trained using the training set due to Kobe earthquake with $10 \%$ amplitude is also very efficient for the earthquake record with different amplitude.

As a last example, the performance of the neuro-controller trained above for different kind of earthquake record, is demonstrated. Not loss the generality, the numerical simulation is carried out 
for the load case when structure-actuator coupled system is subjected to the El-Centro earthquake with 20\% amplitude. The results are illustrated in Fig. 22 and Fig. 23.

Although there are a lot of differences in the peak, shape, and amplitude between the EL-Centro earthquake with $20 \%$ amplitude and the Kobe earthquake with $10 \%$ amplitude which is used to train the neuro-controller, the numerical simulations indicate that the neuro-controller is as effective in controlling the coupled structure when it is subjected to El-Centro earthquake record, as it is in controlling the structure when the structure is subjected to the earthquake record which the neuro-controller was trained on. This demonstrates the fact that the neuro-controller learns to control the motion of the coupled structure, regardless of the source of excitation if actuators with enough capability are designed.

\section{CONCLUSION}

A new method of localized active control for large-scale or complex structures using artificial neural networks is proposed in this paper. In this proposed control method, a neuro-controller replaces the control algorithm of conventional localized control method, which is usually based on the method of model reduction and generalized singular linear quadratic algorithm. And the control-structure interaction (CSI) and dynamics of typical electro-hydraulic actuators are considered. Throughout the numerical simulations the following conclusions may be made:

(1) A neural emulator network can be trained to forecast the dynamic response in localized areas of large-scale structure according to partial information (displacement and acceleration) from each localized area, earthquake record and control signals. In other words, localized identification can be carried out by multi-layer neural network when the controlstructure interaction and dynamic of actuator is considered.

(2) During the procedure of vibration control of coupled system under earthquake excitation, based on the trained neural emulator network, a neuro-controller can also be trained to decide the necessary control signals according to partial information from each localized area of coupled system. The dynamic response of each localized area can be controlled by the actuator located in the area with neuro-controller.

(3) To large-scale or complex structures, localized vibration control by using multi-layer neural network is a promising method under earthquake when dynamics of actuator is considered.
In localized vibration control problem, less sensors and less computation cost are necessary. Localized vibration control is an economical method for large-scale or complex structure. And on the other hand, because the scale of neural network used in localized vibration control is small, the procedure of training the neural emulator network and calculating the control signals are less time-consuming. This kind of advantage is very useful for on-time control.

(4) The method of localized vibration control using multi-layer neural network is adaptable for earthquakes different from it used for training the neuro-controller. The learning capabilities of the neuro-controller make it an adaptive controller.

\section{REFERENCES}

1) Leipholtz, H. H. and Abdel-Rohman, M.: Control of Structures. Martinus Nijhoff Publishers, The Hague, The Netherlands, 1986.

2) Soong, T.T.: Active structural control, Longman Scientific and Technical, 1990

3) Spencer Jr., B.F., Dyke, S.J. and Deoskar, H.S.: Benchmark Problems in Structural Control: Part I- Active Mass Driver System, Earthquake Engineering and Structural Dynamics, 27, pp.1127-1139, 1998.

4) Spencer Jr., B.F., Dyke, S.J. and Deoskar, H.S.: Benchmark Problems in Structural Control: Part II- Active Tendon System, Earthquake Engineering and Structural Dynamics, 27, pp.1141-1147, 1998.

5) Ghaboussi J., and Joghatatie, A.: Active control of structures using neural networks, Journal of Engineering Mechanics, ASCE, 121(4), pp.555-567, April 1995.

6) Chen, H.M., Tsai, K.H., Qi, G.Z., Yang, J.C.S. and Amiini, F.: Neural network for structure control, Journal of Computing in Civil Engineering, Vol.9, No.2, April, 1995.

7) $\mathrm{Xu}, \mathrm{B} ., \mathrm{Wu}, \mathrm{Z} . S$. and Yokoyama, K.: Adaptive vibration control of structure-AMD coupled system using multi-layer neural networks, Journal of Applied Mechanics, JSCE, Vol.3, pp.427-348, September 2000.

8) Hannsen Su, J. and Ruckman, C.E.: An isolated structural system using localized vibration control, Journal of Intelligent Material Systems and Structures, Vol.6, pp.801-808, November 1995.

9) $\mathrm{Xu}, \mathrm{B} ., \mathrm{Wu}, \mathrm{Z} . \mathrm{S}$. and Yokoyama, K: Adaptive localized vibration control of large-scale or complex structures using multi-layer neural network, Proceeding of the Seventh East Asia-Pacific Conference on Structural Engineering and Construction, Japan, August 1999.

10) Sato, T. and Kikukawa, M.: A linear algorithm to identify the non-linear structural system equations, Journal of Structural Mechanics and Earthquake Engineering, JSCE, No.584/I-42, pp.175-184, Jan. 1998. (In Japanese)

11) $\mathrm{Xu}, \mathrm{B} ., \mathrm{Wu}, \mathrm{Z} . \mathrm{S}$. and Yokoyama, K.: Decentralized identification of large-scale structure-AMD coupled system using multi-layer neural networks, Transactions of Japan Society of Computational Engineering and Science, JSCES, Vol.2, pp. 187-197, May 2000

12) Bani-hani, K., Ghaboussi, J. and Schneider, S.P.: Experimental study of identification and control of structures using neural network, Part 1: Identification, Earthquake Engineering and Structural Dynamics, ASCE, 28, pp.995-1018, 1999.

13) Bani-hani, K., Ghaboussi, J. and Schneider, S.P.: Experimental study of identification and control of 
structures using neural network, Part 2: Control, Earthquake Engineering and Structural Dynamics, ASCE, 28, pp.1019-1039, 1999.

14) Bani-hani, K. and Ghaboussi, J.: Nonlinear Structural Control Using Neural Networks, Journal of Engineering Mechanics, ASCE, 124(3), pp.319-327, 1998.

15) Kumagai, T., Hashimoto, R. Wada, M., Tanaka, M. and Yoshida, Y.: Control of an active mass damper using a neural network, Journal of JSME(C), 59(564), pp.41-47, August 1993. (In Japanese)
16) Sato, T., Toki, K. and Hashimoto M.: Active structural response control with self-learning mechanism, Journal of Structural Mechanics and Earthquake Engineering, JSCE, No. 471/1-24, pp.115-124, July 1993. (In Japanese)

17) Hagiwara, M.: Theoretical derivation of a momentum term in back-propagation, International Joint Conference on Neural Networks, Vol.1, pp.682-686, Baltimore, MD, 1992.

(Received December 6, 1999)

\title{
階層型ニューラルネットワークによる構造一アクチュエータ連成システム の局所化制御に関する研究
}

\author{
許 斌・吳 智深・横山 功一・原田 隆郎
}

地震時における安全性の向上を目的とした構造物のアクティブ振動制御に関する研究とその実用化が大 いに期待されている. 本論文では, 階層型ニューラルネットワークを用いて, アクチュエータの動力特性 を生かした構造ーアクチュエータ連成システム構築し, 構造システムの局所化同定及び局所化制御領域の 応答值の予測, 更に実現可能な局所化制御信号計算方法等の問題について検討した. そして, 大型で複雑 な制御対象にも適用できるように，局所化システムの情報によって適切な制御信号を出せるような制御シ ステムに発展させた. 数值シミュレーションの結果, 状態予測および制御信号の算定において精度の高い 結果が得られ、システムの有効性とロバストな適応制御アルゴリズムが確認できた. 